\title{
Combined bilateral giant coronary aneurysm and coronary fistula to coronary sinus
}

\author{
Hiroya Takafuji, Shinobu Hosokawa, Riyo Ogura, Yoshikazu Hiasa \\ Department of Cardiology, Tokushima Red Cross Hospital, Tokushima, Japan
}

The prevalence of giant coronary aneurysm and fistula in coronary angiogram is $0.02-0.2 \%$ and $0.2-2 \%$, respectively. Consequently, combined giant coronary aneurysm and fistula are extremely rare abnormalities of the heart.

A 65-year-old male was coincidentally demonstrated to have abnormalities surrounding the heart by chest computed tomography. The patient had continued medical follow-up because he was asymptomatic, but ejection fraction and left ventricle size had gradually worsened over the years. Transthoracic echocardiography showed multiple abnormal cavities in both the right and left atria (Fig. 1A). Color Doppler in transesophageal echocardiography showed continuous color signal and flow in the abnormal cavity (Fig. 1B, C). In addition, coronary computed tomography revealed a giant bilateral coronary aneurysm (Fig. 1D). Coronary angiography confirmed a huge aneurysm at the left circumflex and right coronary artery with a fistula communicating with the coronary sinus (Fig. 1E, F). The pulmonary blood flow to systemic blood flow $(\mathrm{Qp} / \mathrm{Qs})$ ratio measured using right heart catheterization was 1.63 . The left to right shunt ratio was $39 \%$. Hence, radical surgery was performed by closure of the arterio-venous fistula and ligation of the bilateral abnormal arteries without a coronary artery bypass graft operation.

Coronary aneurysm and fistula are associated with increased risk of cardiac events, such as cardiac rupture, coronary ischemia, arrhythmia, and thromboembolism. Therefore, it is necessary to establish an immediate diagnosis using multimodality imaging and initiate treatment. If the patient is previously asymptomatic, regular follow-up to check cardiac function and cardiac load is crucial in determining the timing of surgical intervention.

Conflict of interest: None declared

Address for correspondence: Hiroya Takafuji, MD, 103 Irinokuchi, Komatsushima-cho, Komatsushima, Tokushima 773-8502, Japan, tel: +81-885-32-2555, fax: +81-885-32-6350, e-mail: takafuji@tokushima-med.jrc.or.jp 


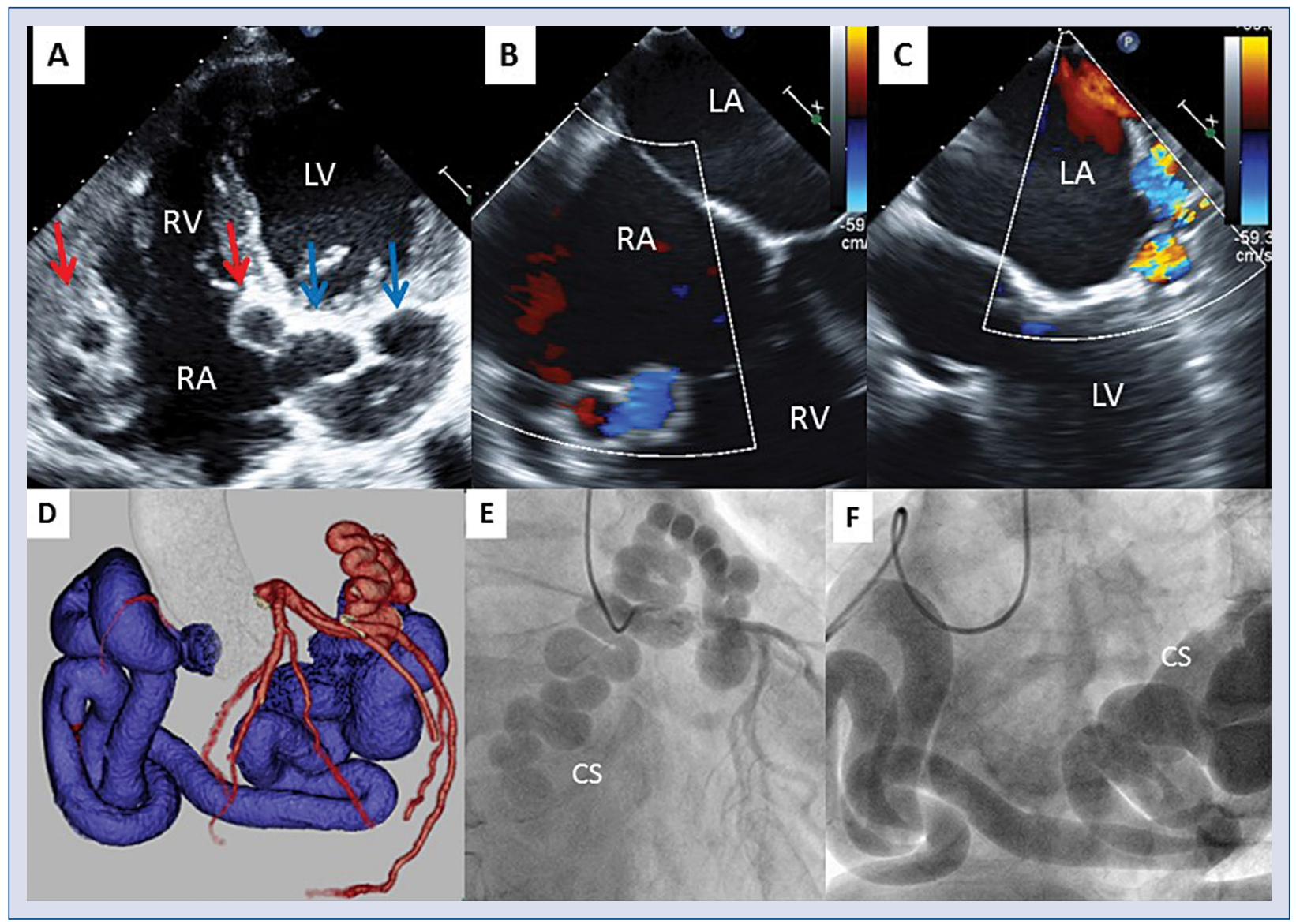

Figure 1. A. Transthoracic echocardiography showed multiple abnormal cavities at both the right atrium (RA; red arrow) and left atrium (LA; blue arrow); B, C. Transesophageal echocardiography with color Doppler revealed abnormal flow in cavities around the RA and LA; $\mathbf{D}$. Three-dimensional reconstruction of coronary computed tomography showed a bilateral giant coronary aneurysm; E, F. Coronary angiography revealed a combined giant coronary artery aneurysm with fistula communication to the coronary sinus (CS); LV — left ventricle; RV — right ventricle. 